\title{
Ordering in lattice gases at low temperature
}

\author{
Charles Radin \\ Mathematics Department, University of Texas at Austin, Austin, TX 78712, USA
}

Received 25 April 1988, in final form 1 September 1988

\begin{abstract}
We show that for generic short-range classical lattice gas models the third law of thermodynamics holds-entropy goes to zero with temperature.
\end{abstract}

The most fundamental aspect of low-temperature matter is its highly ordered (crystalline) structure, which distinguishes it from the fluid state and is necessary for most of the characteristic features of solids.

Attempts to discover the causes of this order constitute, in one way or another, one of the main themes of solid state physics (see [1]) and have been, to date, largely unsuccessful. At the most basic level one models equilibrium matter by statistical mechanics, which means by a particle system in a configuration which minimises the free energy. It is expected that, at low temperature, in order to minimise the free energy one must in fact minimise the energy, and to minimise the energy the particle system must be in a highly ordered (crystalline) configuration. It is this last part of the conventional intuition, whereby low energy forces configurations to have crystalline order, that the main problem lies; there is not even the vaguest, most intuitive argument as to why or by what mechanism this forcing is supposed to take place. For an extensive review of this problem see [2].

A new line of development has opened in this 'crystal problem' (the attempt to understand why low-energy configurations must have crystalline order) in which the new feature is that the role of crystalline order is questioned. More specifically, using classical lattice gas models it has recently been proved $[3,4]$ that for generic short-range interactions the ground state has long-range order but is not crystalline. (By long-range order we mean that two-particle correlation functions do not cluster, or intuitively that portions of the configuration in regions separated arbitrarily far apart are statistically dependent.) The main point of these papers is the strong suggestion that it is appropriate to aim at proving that low energy implies not crystalline order but only something weaker; for example long-range order.

The aim of this paper is twofold. First we show that the special property of configurations used in [3], from which long-range order was proven to follow, is of more significance than was realised; from it also follows the vanishing of the entropy, a somewhat different measure of order. Second, the rest of [3] then implies that the third law of thermodynamics holds for generic interactions.

The idea in [3] from which we start is the following. We call a configuration 'net-ordered' if, given any $\varepsilon>0$, there are three linearly independent vectors $\boldsymbol{d}_{j}(\varepsilon), j=$ $1,2,3$, such that, except for a fraction of the configuration less than $\varepsilon$, the configuration is locally invariant under translation by $\boldsymbol{d}_{j}(\varepsilon)$. This is a little vague; for lattice gas models we make the definition precise by replacing the configuration is locally 
invariant' by 'the state of the configuration at each site is invariant'. It is necessary at this point to make precise exactly what we mean by classical lattice gas models.

In classical lattice gas models one considers a field, $\phi$, on the three-dimensional simple cubic lattice, $\boldsymbol{Z}^{3}$, with finitely many possible values (let $S$ denote the number) at each lattice site, each value interpreted as the occupation status of the site; one value is reserved to mean 'empty' and the others represent different internal states of a single particle. We will refer to a set of field variables for all sites of $\boldsymbol{Z}^{3}$ (or a subset if so indicated) as a 'configuration'. By a 'ground-state distribution' we mean a limit, as temperature goes to zero, of the translation-invariant grand canonical probability distribution on the set of all configurations, and by a 'ground-state configuration' we mean any configuration in support of the ground-state distribution.

Next we wish to prove some consequences that follow when one knows that a configuration is net-ordered. To do this we restate the definition of a net-ordered configuration in terms of the restrictions of the configuration to large finite cubes of sites in $\boldsymbol{Z}^{3}$, as follows. A configuration is net-ordered if, given $\varepsilon>0$ and any cube $\Lambda$ containing $M N$ sites and with sides perpendicular to the coordinate directions, where $M=1 / \varepsilon$ and $N \gg M$, the restriction, $\gamma$, of the configuration to $\Lambda$ satisfies the following condition: except for $X \leqslant N$ of the sites of $\Lambda$ the state at each site agrees with that at those three sites (assumed to be again in $\Lambda$ ) at some distance (denoted $r(M)$ ) along the three axes in the positive directions, $\boldsymbol{e}_{i}$. (Intuitively, $N$ measures the size of $\Lambda$ and $\varepsilon=1 / M$ is an upper bound on the fraction of exceptional sites; the state at each of the other (non-exceptional) sites is invariant under translation in three directions by the 'period' $r(M)$.)

In order to estimate the entropy of a model we will establish an upper bound on how many such restrictions $\gamma$ are possible. Fix some $\gamma$. In the above 'condition' call the $X$ exceptional sites 'bad' and the remaining ones 'good'. (The union of the good and bad sets is $\Lambda$, and so consists of $M N$ sites.) Define a 'component' to be a maximally connected set of good sites, i.e. a maximal set of good sites, any two of which can be connected by a sequence of steps, of the same fixed length $r(M)$ parallel to any of the three axes, between sites in the set. Note that if $s$ belongs to some component then either $s+r(M) e_{1}$ also belongs to that component, or it is a bad site. It follows then that for each component there is at least one bad site of the form $s+r(M) e_{1}$ for some site $s$ in the component, which then implies that $\gamma$ has at most $X$ components.

We are interested in the number of possible configurations $\gamma$ on $\Lambda$ which satisfy the condition. To get an upper bound first note, using the above, that there are at most $S^{2 X}$ which have their bad sites in the same $X$ places. Second, note that there are $(N M) ! /[X !(N M-X) !]$ ways to place the $X$ bad sites in $\Lambda$. So the number of configurations is at most

$$
\Gamma(M, N)=\sum_{X=0}^{N} \frac{(N M) ! S^{2 X}}{(N M-X) ! X !}<\frac{(N+1)(N M) ! S^{2 N}}{[(M-1) N] ! N !} .
$$

We will show next that

$$
\lim _{M, N \rightarrow \infty} \frac{\log \Gamma(M, N)}{M N}=0 .
$$

From Stirling's approximation, $\log (z !)=\left(z+\frac{1}{2}\right) \log (z)-z+\mathrm{O}(1)$ where $\mathrm{O}(1)$ stands for something bounded as $z \rightarrow \infty$. So for fixed $M$, as $N \rightarrow \infty$ we have 


$$
\begin{aligned}
& \log \left(\frac{(N M) !}{[(M-1) N] ! N !}\right) \\
& \quad=N M \log \left(\frac{M}{(M-1)}\right)+\frac{1}{2} \log \left(\frac{M}{(M-1)}\right)+N \log (M-1)-\frac{1}{2} \log (N)+o(1)
\end{aligned}
$$

which shows that

$$
\lim _{M, N \rightarrow \infty} \frac{\log \Gamma(M, N)}{M N}=0 .
$$

Now it is an immediate consequence of proposition 3 in [5] that

$$
\lim _{M, N \rightarrow \infty} \frac{\log \Gamma(M, N)}{M N}
$$

is the zero-temperature limit of the entropy. So, if one can prove for some interaction that all of its ground-state configurations have the property of being net-ordered in the above sense, then the above argument shows that for that model the entropy goes to zero with temperature, i.e. the model satisfies the third law of thermodynamics. Since Miękisz proved [3] that among short-range lattice models a generic set of interactions are net-ordered, it follows that among short-range lattice gas models the third law is satisfied generically. Perhaps of more importance, this indicates that the property of configurations that they be net-ordered is technically a very useful property.

We summarise our results in the following theorem.

Theorem. (a) If the ground-state configurations of an interaction are all net-ordered, for that model entropy goes to zero with temperature. (b) Among short-range lattice gas models the ground-state configurations of generic interactions are all net-ordered and therefore the third law of thermodynamics holds generically.

\section{Acknowledgments}

It is a pleasure to acknowledge useful conversations with Larry Schulman and Dennis Sullivan. This research was supported in part by NSF grant no DMS-8701616.

\section{References}

[1] Anderson P W 1984 Basic Notions of Condensed Matter Physics (New York: Benjamin)

[2] Radin C 1987 Int. J. Mod. Phys. B 11157

[3] Miȩkisz J 1988 J. Phys. A: Math. Gen. 21 L529

[4] Miȩkisz J and Radin C 1989 Phys, Rev. B in press

[5] Aizenman M and Lieb E H 1981 J. Stat. Phys. 24279 